\title{
PENSAMENTO COMPUTACIONAL NO ENSINO MÉDIO: PRÁTICAS MEDIADORAS UTILIZANDO A LINGUAGEM SCRATCH
}

\author{
Leonardo Poloni - UCS - lpoloni@ucs.br \\ Eliana Maria do Sacramento Soares - UCS - emsoares@ucs.br \\ Carine G. Webber - UCS - cgwebber@ucs.br
}

Resumo. O pensamento computacional envolve habilidades que procuram aprofundar o conhecimento e as capacidades dos estudantes. Apresenta-se, neste artigo, um estudo cujo foco foi identificar formas de mediação possibilitadas pela linguagem Scratch no processo de ensino-aprendizagem de programação. O quadro teórico baseou-se na teoria sociointeracionista de Vigotski. O percurso metodológico foi delineado por um estudo de caso, baseado em uma oficina de programação. $O$ resultado permitiu inferir que o ambiente Scratch tem potencial para mediar o aprendizado de programação. Contudo, o professor precisa também atuar como mediador criando estratégias e intervenções potenciais para auxiliar o estudante.

Palavras chaves: Ensino Médio. Tecnologia Educacional. Linguagem de Programação. Pensamento Lógico.

\section{COMPUTATIONAL THINKING IN HIGH SCHOOL: MEDIATED PRACTICES USING SCRATCH PROGRAMMING LANGUAGE}

\begin{abstract}
Computational thinking look for students' knowledge and skills continuous improvement. In this article, we propose a study whose focus was to identify forms of mediation through the Scratch language during a computer programming learning process. The theoretical framework was based on Vigotski's social interactionist theory. The methodological approach was outlined by a case study, based on a programming workshop. The result allowed the conclusion that Scratch environment has the potential to mediate programming learning. However, the teacher also needs to act as a mediator by creating strategies and potential interventions in order to help students.
\end{abstract}

Keywords: High School. Educational Technology. Programming Language. Logical Thinking 


\section{Introdução}

Como preconizam autores, como França e Amaral (2013), e a própria BNCC (Brasil, 2018) precisamos integrar aspectos básicos da Ciência da Computação na formação dos estudantes. Algumas das demandas da prática educativa estão relacionadas à capacidade de resolução de problemas de forma crítica e utilizando o pensamento lógico dedutivo. Essas capacidades são relevantes em diversas áreas e relacionadas a diversos conteúdos, em especial os que dizem respeito ao pensamento computacional.

O pensamento computacional compreende um conjunto de habilidades relacionadas à forma com que os seres humanos podem usar os computadores para resolver problemas. Embora o conceito de pensamento computacional tenha sido abordado previamente por Papert (2008), quando ele se refere às habilidades mobilizadas pelos estudantes durante o uso dos computadores e jogos, foi Wing (2006) quem o popularizou. Essa autora apresentou o pensamento computacional como um conjunto de habilidades básicas e necessárias, importantes de serem desenvolvidas por todos, independentemente da área de estudo ou atuação.

Países como Estados Unidos, Espanha, Reino Unido e outros vêm alterando seus currículos escolares, pensando na formação de cidadãos capazes de atuar em uma sociedade tecnológica. Essa alteração consiste em inserir o pensamento computacional de forma gradual na formação dos estudantes, incluindo linguagens de programação para estudantes a partir dos cinco anos. No Brasil, o aprendizado de programação também está ganhando mais espaço na grade curricular ou extracurricular das escolas.

Alinhado a este contexto, o presente trabalho apresenta um recorte de uma dissertação de mestrado que se propôs a analisar se a linguagem Scratch tem potencial para ser elemento mediador da aprendizagem, na perspectiva vigotskiana. O processo de mediação, por meio de instrumentos e signos, é para Vigotski fundamental para o desenvolvimento das funções psicológicas superiores (Martins e Moser, 2012). Para entender como isso pode ser possível foi desenvolvida a presente pesquisa.

O presente artigo está organizado em quatro seções. A seção dois apresenta uma breve revisão bibliográfica de autores que fundamentam este trabalho. A seção três descreve o estudo de caso e o método de análise dos resultados. Na seção quatro apresenta-se como transcorreu o estudo de caso e as avaliações realizadas. Por fim, são apresentadas as considerações finais sobre o trabalho.

\section{Fundamentação Teórica: Pensamento Computacional e Vigotski}

O pensamento computacional é um conceito abrangente que compreende habilidades que podem ser desenvolvidas pela programação. A primeira destas habilidades esta associada à capacidade de compreender um problema, interpretando-o em termos de componentes ou partes constituintes. A forma como os componentes se combinam, integram e compõe sua solução permite avaliar a habilidade denominada de decomposição de problemas em subproblemas. Em seguida, as habilidades de abstração e reconhecimento de padrões são as que possibilitam que as partes mais importantes de um problema sejam priorizadas e tratadas de acordo com padrões previamente aprendidos e assim reutilizados. Em termos de estruturação da resolução de um problema, as habilidades de pensamento sequencial, condicional e paralelo indicam a organização lógica e analítica do indivíduo. Ela envolve ainda formas para aprimorar os 
métodos de resolução existentes, buscando melhorar a eficiência global de uma solução, estimulando assim o pensamento lógico e dedutivo (CSTA, 2011).

Resnick e outros autores (2009) argumentam que a preparação das crianças e jovens para atuarem na sociedade tecnológica depende do desenvolvimento de habilidades que os permitam projetar tecnologias, criar artefatos e combinar recursos digitais de forma original. Para isso, defendem que somente com um aprofundamento no conhecimento e domínio das bases computacionais, em especial na programação de computadores, pode-se superar este desafio.

Recentemente uma série de softwares educativos visuais, e inspirados em jogos, tem surgido para incentivar o ensino de programação. Um dos softwares mais utilizados mundialmente é a linguagem de programação Scratch. Ela foi desenvolvida a partir de pesquisas e aperfeiçoamentos das linguagens e ambientes de programação para crianças (em especial o LOGO) por Resnick (2009). A partir das suas funcionalidades, ela possibilita a criação de histórias, animações, simulações e jogos por meio da programação em blocos visuais e retroação automática.

Diversos experimentos utilizando a linguagem Scratch tem evidenciado seu potencial para promover o desenvolvimento do pensamento computacional. Destaca-se aqui alguns trabalhos que inserem o ensino da programação utilizando Scratch na educação básica. Scaico e outros autores (2013) apresentaram uma experiência realizada com ensino de programação em escolas públicas, promovendo uma olimpíada de programação. De forma similar, Wangenheim e outros autores (2014) propuseram uma unidade instrucional para o ensino de programação usando o software Scratch para uma turma de primeiro ano do ensino médio. Esses autores reportam que os experimentos e as avaliações realizadas evidenciam a facilidade no uso do ambiente bem como sua efetividade em termos de aprendizagem de conceitos básicos de programação. Aono e outros (2017) apresentam um estudo utilizando o Scratch, onde foi proposto a construção de um jogo. Por meio da construção do jogo, elementos de programação foram aprendidos. Observa-se também o uso do Scratch em conjunto com disciplinas tais como História e Estudos Sociais (ALVES et.al., 2016).

As recomendações dos autores desses estudos permitem inferir que práticas pedagógicas integrando o Scratch tornam o processo de aprendizagem de programação divertido, despertando o interesse e motivação dos estudantes para essa área de conhecimento. Ainda assim, programar constitui uma tarefa complexa para a qual os estudantes necessitam de elementos de mediação. Evidências indicam que uma das causas da dificuldade em aprender-se a programar está na falta de habilidade para resolver problemas matemáticos e lógicos (Gomes et al., 2006).

Para Kumar (2005), para que um estudante entenda um problema, ele depende da intervenção e orientação do professor. Uma forma eficiente de auxiliar o estudante em dificuldade consiste no professor construir, conjuntamente com o estudante, uma estrutura inicial, base da solução do problema. A partir deste ponto, estudos mostram que uma retroação imediata auxilia o aprendizado (Schulze, 1989, Kumar, 2005), informando ao estudante onde estão seus erros e indicando as melhorias. A execução de um algoritmo passo a passo é outra forma de um sistema auxiliar o estudante a compreender o funcionamento de uma solução. Observa-se na prática que, embora a retroação por parte dos sistemas seja útil, ela sozinha não resolve as dificuldades na 
aprendizagem de programação, pois ela vai além do uso e manipulação de símbolos.

Percebe-se assim a necessidade de um aporte teórico que permita compreender o papel das linguagens de programação, no caso de uma linguagem com recursos visuais como o Scratch, e no processo de aprendizagem das habilidades do pensamento computacional. Neste contexto, a teoria de Vigotski preconiza que a aprendizagem é uma experiência social, mediada pela interação entre a linguagem e a ação realizada.

Para Vigotski é importante identificar o estado de desenvolvimento mental dos estudantes, reconhecendo o nível real (tarefas que o aluno realiza sozinho) e a zona desenvolvimento proximal (tarefas que ele está potencialmente apto a aprender desde que seja orientado). A mediação pedagógica na zona de desenvolvimento proximal (ZDP) pode conduzir a avanços cognitivos que de outra forma poderiam não se concretizar (GONÇALVEZ, 2004). Além disso, Vigotski (2007) percebeu que o desenvolvimento os estudantes poderia ser melhor avaliado quando eles trabalhavam em conjunto, reconhecendo que o conhecimento é o resultado da interação social.

No contexto educativo, a identificação da ZDP possibilita ao professor realizar intervenções com potencial para levar o estudante a estabelecer conexões entre o novo conhecimento em construção e os conceitos prévios. Vigotski (2007) explica que a conversão de ações e interações sociais, externas ao sujeito, em funções mentais superiores é mediada por ações ou instrumentos, podendo conduzir a significação.

\section{Material e Métodos}

O percurso metodológico desta pesquisa foi delineado por um estudo de caso, criado a partir de uma oficina de introdução à programação de computadores para cinco estudantes do primeiro ano do Ensino Médio Técnico de uma instituição federal de ensino. A oficina foi oferecida aos estudantes (denominados E1, E2, E3, E4 e E5), que voluntariamente demonstraram interesse e participaram da oficina.

A referida pesquisa buscou analisar como o Scratch pode mediar a aprendizagem de programação no Ensino Médio com vistas ao desenvolvimento do pensamento computacional, a partir dos conceitos de mediação e aprendizagem, apoiado pelas três dimensões do framework de Brennan e Resnick (2012). O framework foi desenvolvido com o objetivo de permitir compreender quais competências do pensamento computacional podem ser exploradas com o uso de uma linguagem de introdução à programação como o Scratch. Esse framework está dividido em três dimensões: conceitos computacionais, práticas computacionais e perspectivas computacionais (BRENNAN e RESNICK, 2012; MORENO-LEÓN e ROBLES, 2015).

A escolha da linguagem o Scratch para essa oficina levou em consideração a questão de ser uma ferramenta de programação educativa gratuita e de possuir uma versão que pode ser acessada a partir do navegador da internet. O corpus foi constituído pelos cadernos de reflexão dos estudantes, pelos programas criados pelos estudantes para resolver cada tarefa, pelas anotações do pesquisador e por questionário pós-oficina. A análise do corpus, na busca de compreender e explicar o contexto dos estudantes, atuando na oficina e utilizando o Scratch, seguiu as seguintes etapas: a) tabulação dos dados dos questionários pré-oficina; b)submissão dos programas criados pelos estudantes para resolver cada tarefa à análise do Dr. Scratch; c)análise manual dos programas criados pelos estudantes com base no framework de Brennan e Resnick 
(2012); e, d) análise conjunta dos cadernos de reflexão dos estudantes, das anotações do pesquisador e dos questionários pós-oficina, articulando com os resultados das etapas anteriores para construir a resposta à pergunta de pesquisa.

O planejamento e execução da oficina seguiu a proposta de Vigotski, propondo ações que levassem os estudantes a criarem seu caminho de exploração, a partir de tarefas desafiadoras, envolvendo os conceitos de programação. Seguindo a perspectiva vigotskiana, o professor precisa realizar intervenções para que as atividades produzidas no Scratch sejam significadas pelos estudantes. Ainda, a mediação deve ser realizada na ZDP do estudante, que pode ser reconhecida pela forma como ele interage, expõe suas dúvidas, erros e como procede nas atividades.

No decorrer da oficina, cada participante produziu em média quatro programas, os quais foram enviados ao professor.. A oficina foi realizada semanalmente, em sete encontros, de 2 horas (totalizando 14 horas). Cada estudante registrou suas percepções sobre a oficina e respondeu a questões, com o objetivo de externar suas percepções quanto ao uso do Scratch, explicitar as suas dificuldades e descobertas, além de sistematizar o processo de resolução das tarefas propostas.

\section{Resultados e Discussão}

\subsection{Análise do corpus}

$\mathrm{O}$ corpus de pesquisa foi analisado buscando identificar e compreender as possibilidades de mediação do Scratch no processo de aprendizagem de programação com estudantes do Ensino Médio, tendo como principal norteador a teoria vigotskiana e os princípios do pensamento computacional.

Primeiramente, os programas foram submetidos à análise automatizada realizada pelo software Dr. Scratch, que inspeciona os projetos sob a perspectiva do desenvolvimento do pensamento computacional. Ele opera identificando níveis (básico, desenvolvido e proficiente) e atribuindo pontuação correspondente quanto ao emprego de 7 conceitos-alvo: abstração, pensamento lógico, sincronização, paralelismo, controle de fluxo, interatividade com o usuário e representação dos dados. Cada conceito-alvo pode ser avaliado em nível básico ( 1 ponto), desenvolvido ( 2 pontos) ou proficiente ( 3 pontos), segundo a escolha de instruções do estudante.

A Figura 1 ilustra um relatório de análise elaborado pelo Dr. Scratch, o qual foi gerado a partir do primeiro projeto de um dos participantes da oficina. Em sua análise, o Dr. Scratch atribuiu 7 pontos a esse projeto (de um total de 21), classificando o projeto no nível Básico. A pontuação obtida reflete o fato de que nem todos os conceitos-alvo foram mobilizados ou aprofundados na construção do estudante.

No decorrer da oficina, os participantes criaram dezenove projetos que, ao serem submetidos à avaliação do Dr. Scratch, receberam as pontuações representadas no Gráfico 1. Em relação ao primeiro projeto, sobressai a pontuação obtida pelo participante E1 que atingiu 13 pontos. É possível observar que a maioria dos estudantes apresentou uma evolução em relação à pontuação obtida em cada projeto. Houve casos em que o estudante optou por não entregar um projeto, o que justifica os dados faltantes. 
Figura 1 - Projeto empregando o pensamento computacional em nível básico.

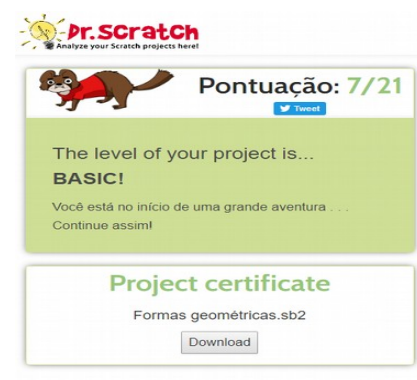

Fonte: criado pelo autor.

Ao analisar os programas, o Dr. Scratch avalia os comandos e estruturas empregados, não levando em consideração o enunciado da tarefa. Por esse motivo, decidiu-se realizar uma segunda análise, avaliando e testando manualmente cada projeto desenvolvido, na busca por reconhecer como o estudante estruturou sua lógica para elaborar a solução de cada tarefa. Com isso buscou-se identificar quais conceitos de programação foram empregados, que funcionalidades foram implementadas, além de verificar erros e dificuldades do estudante no desenvolvimento dos projetos.

Além disso, foram utilizadas as anotações realizadas pelo professor no decorrer da oficina, os registros realizados pelos participantes durante as aulas, bem como as respostas dadas por eles ao questionário pós-oficina. Os resultados obtidos com a segunda análise trouxeram alguns indicativos sobre o percurso do estudante ao longo da oficina. Ela permitiu uma compreensão minuciosa do desenvolvimento dos estudantes, identificando por exemplo que o estudante E4 apresentou avanços na resolução de problemas entre as tarefas (contrariando a análise automática realizada pelo Dr. Scratch, que pontuou pela presença de instruções lógicas). De fato, o estudante E4 apresentou estruturação lógica e sequencial dos comandos aprimorada no segundo projeto.

Gráfico 1- Pontuação atribuída pelo Dr. Scratch a cada projeto analisado.

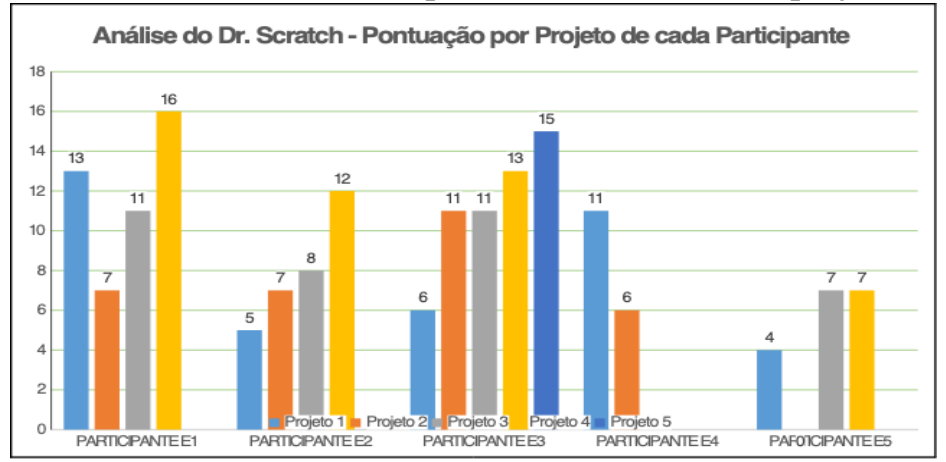

Fonte: os autores.

Essa diferença entre a primeira e a segunda análise também foi evidente com relação ao participante E1, onde na análise do projeto 2 o Dr. Scratch atribuiu 7 pontos, e considerou que o programa atingiu apenas o primeiro nível, denominado de "básico" e, em comparação com o primeiro projeto, mostrou uma queda considerável na pontuação resultando na queda de nível também. Por outro lado, na segunda análise, levando em consideração o problema proposto, conclui-se que o projeto ficou coerente 
com o que foi sugerido, sendo que o estudante evidenciou a exploração de outros recursos do Scratch, bem como teve um cuidado com a inicialização das variáveis, o que foi pouco observado no primeiro projeto.

O oposto também ocorreu, ou seja, o Dr. Scratch atribuir uma pontuação superior ao que foi observado na segunda análise. Um exemplo disso ocorreu com a análise do projeto 2 do participante E3. Quando comparado ao primeiro projeto desse estudante, a avaliação feita pelo $D r$. Scratch mostrou um grande aumento na pontuação, resultando na mudança para o segundo nível. Já na segunda análise, levando em consideração a proposta do participante para essa atividade, observa-se que o estudante atingiu de forma básica seus objetivos, mostrando avanço na utilização de alguns recursos. Entretanto, ficou incompleto e apresentou objetos e códigos que não são utilizados para nada, também denominado de "código morto",

A avaliação dos projetos possibilitou traçar o percurso dos estudantes no decorrer da oficina, o qual revelou uma evolução dos participantes no que diz respeito à aprendizagem dos conceitos relacionados à programação de computadores. Além disso, evidenciou o emprego de princípios do pensamento computacional, independentemente do nível de ensino e dos conhecimentos prévios de cada participante. Essa avaliação não buscou comparar o desempenho entre os estudantes, mas sim a evolução de cada um.

De forma geral, foi possível observar que os participantes evoluíram, tanto no grau de complexidade dos elementos e blocos de comandos que usaram para compor os projetos, quanto nas relações lógicas utilizadas na construção do algoritmo por trás da solução apresentada. Segundo Resnick (2009), a descoberta, na experiência lúdica, de conceitos matemáticos e computacionais, possibilitam o treinamento do pensamento sistêmico, necessário para o século XXI. Nesse sentido, os participantes da oficina mostraram interesse e curiosidade em explorar e aprender a utilizar os recursos do Scratch. Observou-se que os participantes conseguiram assumir o papel de autores, programaram, criaram e compartilharam suas criações, pensando criativamente.

\subsection{Análise do papel do professor}

Outro aspecto que aparece como resultado da análise do corpus é a importância do professor, cujo papel necessita ser redimensionado. Na efetivação da oficina, o professor precisou sair do lugar do discurso e ir para o lugar da mediação. Para isso, ele necessitou aprender a atuar como mediador, buscando avaliar o que os estudantes haviam compreendido, qual era sua ZDP, para então encontrar táticas de atuação. Foi preciso autocontrole para que não desse as respostas de imediato, para que não apontasse os erros e não dissesse o que deveria ser feito para corrigi-los.

De acordo com Vigotski (2007), a mediação é uma ação que ocorre no âmbito social por meio de instrumentos, objetos, símbolos, dentre outros elementos. Assim, atuar como mediador implicou que o professor buscasse por estratégias para que cada estudante construísse seu caminho até o conhecimento. Questionamentos, criação de situações-problema, analogias e motivação foram algumas dessas estratégias adotadas.

O professor, seguindo a teoria vigotskiana, ao observar os estudantes consegue fazer intervenções que instiguem, motivem e auxiliem cada um deles, a fim de que 
possam transformar as ações externas realizadas no Scratch em movimentos internos de significação e compreensão. Esses movimentos internos são essenciais para que cada sujeito construa sua rede de conhecimentos, desenvolva sua lógica algorítmica, fazendo relação com os comandos estudados e com isso ocorra o aprendizado de programação.

O questionário aplicado após a oficina também mostrou a percepção que os participantes da oficina tiveram com relação à importância do Scratch e do professor, como mediadores do processo de aprendizagem de programação. Nesse questionário, uma questão abordou a importância de cada elemento (professor, Scratch, material didático, vídeos, e os colegas) no aprendizado de programação e na realização das tarefas propostas. Os resultados atribuíram maior relevância ao professor (pelos 5 estudantes), seguido pelos recursos de retroação do Scratch. Somente depois vieram os recursos didáticos, os colegas e o material em vídeo.

\subsection{Análise das ações realizadas na oficina}

A análise da trajetória dos estudantes considerando a avaliação do Dr. Scratch e do pesquisador, com base no framework de Brennan e Resnick e à luz da teoria vigotskiana, além dos questionários e anotações do pesquisador e dos participantes, possibilitou interpretar e dar sentido aos dados gerados. Pode-se assim, considerar que a linguagem Scratch cumpre seu papel de mediadora do processo de aprendizagem de programação e contribui para o desenvolvimento de princípios do pensamento computacional. Dessa maneira, fornece um sistema de objetos, relações e operadores para expressar e resolver problemas. Entretanto, nesse processo de aprendizagem, o professor tem papel fundamental, tanto na elaboração das situações-problema como na mediação baseada na ZDP de cada participante.

A metodologia empregada na oficina mostrou-se eficaz, pois os participantes se envolveram, demonstrando empenho na exploração do Scratch e na busca por realizar as tarefas. A forma como a oficina foi conduzida possibilitou que cada participante seguisse sua imaginação, exercendo sua criatividade e implementando no ambiente o fruto de sua imaginação. Com isso, os participantes demonstraram motivação e interesse, buscaram auxílio para suas dúvidas em diversos materiais de apoio (vídeos, tutoriais, ajuda do ambiente, comunidade do Scratch), além do professor, o qual buscou não dar respostas diretas às dúvidas e questionamentos, mas sim indicar caminhos.

A metodologia utilizada na oficina teve caráter exploratório, não obrigando os participantes a seguirem um roteiro rígido nem exigindo avaliações ou atribuindo notas aos trabalhos. O foco foi a interação do usuário com o ambiente, tendo o professor como mediador e com a possibilidade de diálogo e auxílio entre os colegas. Esse cenário possibilitou a cada participante seguir seu próprio caminho, atingindo com isso diferentes níveis e características de aprendizado. Isso porque, segundo os estudos de Vigotski (2007), a aprendizagem ocorre à medida que o indivíduo executa operações externas, com o uso de instrumentos e signos, e após reconstrói internamente essas ações, atribuindo significado a elas, em um processo de internalização.

Observou-se que todos os participantes julgaram fundamental o papel do professor e consideraram que a linguagem Scratch lhes ajudou bastante. Esses dois elementos mediadores, atuando de maneira integrada e complementar, forneceram 
possibilidades aos estudantes em desenvolver suas ideias e o conhecimento de programação. Além desses, outro elemento que foi considerado fundamental foi o auxílio e a interação com os colegas. Essa interação propiciou momentos de diálogos e trocas sobre as tarefas, os blocos de comandos ou como melhorar os programas.

A possibilidade de acessar, testar, visualizar como foram desenvolvidos e reaproveitar os programas disponibilizados na comunidade online do Scratch foi considerado fator importante de ajuda por $60 \%$ dos participantes. Os vídeos e demais matérias de apoio tiveram diferentes graus de importância para os estudantes. Cada participante escolheu os recursos que mais lhe agradavam, que possuíam maior familiaridade ou que eram mais conveniente para a situação.

A análise da oficina, ao focar o Scratch como elemento mediador, revelou que, apesar dele possuir um ambiente amigável, estimulante, que motiva e propicia o trabalho autônomo, possibilitando uma iniciação fácil e intuitiva à programação de computadores, necessita de outros elementos nesse processo. Assim como Marques relatou em seus estudos, destaca-se a importância da cooperação, do acompanhamento e da mediação por parte do professor, sem os quais "a produção parece reduzir-se e a evolução não acontece a um ritmo satisfatório" (MARQUES, 2009, p. 32).

Após essas considerações sobre as avaliações do Dr. Scratch e as análises feitas pelo pesquisador, podemos afirmar que os projetos criados pelos estudantes durante as atividades mostraram sua evolução no que diz respeito à aprendizagem. Além disso, evidenciaram o emprego de princípios do pensamento computacional, independentemente do nível de ensino e dos conhecimentos prévios dos participantes.

\section{Conclusões}

Os resultados da análise permitem inferir que os recursos do ambiente Scratch conferem potencial para ser mediador do processo de aprendizagem de programação, fornecendo um sistema de objetos, relações e operadores para expressar e resolver problemas. Sua interface amigável propicia uma iniciação fácil e intuitiva à programação de computadores e contribui para o desenvolvimento de princípios do pensamento computacional. Para Vigotski (2007), os processos internos de desenvolvimento do sujeito estão diretamente relacionados ao ambiente em que estes se desenvolve, bem como ao contexto em que está inserido, envolvendo assim, os indivíduos com os quais compartilha suas experiências. Dessa forma, as práticas vivenciadas pelos estudantes no ambiente Scratch podem ser ponto de partida para que representações mentais sejam desenvolvidas e, com isso, ocorram operações mentais relacionadas à internalização.

Importante destacar que nesse processo o professor tem papel fundamental, tanto na elaboração das situações-problema como na mediação baseada na ZDP dos estudantes. Assim, atuar como mediador implicou na busca por estratégias para que cada estudante construísse seu caminho até o conhecimento. Questionamentos, criação de situações-problema, analogias e motivação foram algumas estratégias adotadas.

Sendo assim, inferimos que esse processo pode ser desencadeado por atividades realizadas no ambiente Scratch, auxiliado por intervenções problematizadoras e instigadoras realizadas pelo professor. Essas são práticas mediadoras com possibilidades 
de levar o estudante a dar um "salto", que é sair da zona potencial de desenvolvimento para a zona de desenvolvimento real. Nessa perspectiva, o uso do Scratch, articulados às intervenções e ações do professor, pode resultar em práticas interativas potencializadoras da aprendizagem de programação.

\section{Referências Bibliográficas}

ALVES, N. C.; RODRIGUES, P.E.; BORGATTO, A.F.; von WANGENHEIM, C.G.; HAUCK, J.C.R. Ensino de Computação de Forma Multidisciplinar em Disciplinas de História no Ensino Fundamental. Revista Brasileira de Informática na Educação, v.24, n.3, p. 31-46, 2016.

AONO, A. H.; RODY, H.V.S.; MUSA, D.L.; PEREIRA, V.A.; ALMEIDA, J. A Utilização do Scratch como Ferramenta no Ensino de Pensamento Computacional para Crianças. In: $\mathbf{2 5}^{\circ}$ Workshop sobre Educação em Computação (WEI 2017). Porto Alegre: SBC, 2017.

BRASIL. Ministério da Educação. Base Nacional Comum Curricular. 2018.

BRENNAN, K.; RESNICK, M. New frameworks for studying and assessing the development of computational thinking. In: Proceedings of the 2012 annual meeting of the American Educational Research Association, Vancouver, Canada, p 1-25.

CSTA - Computer Science Teacher Association; ISTE - International Society for Technology in Education. Operational definition of computational thinking: for k-12 education. 2011. Disponível em: <https://csta.acm.org/Curriculum/sub/CurrFiles/. Acesso em: 20/10/2019.

FRANÇA, R.S.; AMARAL, H.J.C. Proposta metodológica de ensino e avaliação para o desenvolvimento de pensamento computacional com o uso do Scratch. In: XIX Workshop de Informática na Escola, 2013, Campinas.

GOMES, A.; CARMO L.; BIGOTTE, E.; MENDES, A. Mathematics and programming problem solving. In: 3rd E-Learning Conference, Computer Science Education, Coimbra, 2006. KUMAR, A. Generation of problems, answers, grade, and feedback case study of a fully automated tutor. In: J. on Educational Resources in Computing (JERIC), v.5 (issue 3), 2005.

MARTINS, O.B.; MOSER, A. Conceito de mediação em Vygotsky, Leontiev e Wertsch. Revista Intersaberes, vol. 7, n.13, p. 8 - 28, jan. - jun. 2012.

MARQUES, Maria T. P. M. Recuperar o engenho a partir da necessidade, com recurso às tecnologias educativas: contributo do ambiente gráfico de programação Scratch em contexto formal de aprendizagem. Universidade de Lisboa, 2009.

MORENO-LEÓN, J.; ROBLES, G. Analyze your Scratch projects with Dr. Scratch and assess your computational thinking skills. In: Scratch Conference, 2015, p.12-15.

PAPERT, S. A máquina das crianças: repensando a escola na era da informática. Porto Alegre: Artmed, 2008.

RESNICK, M. et al. Scratch: Programming for All. C. of the ACM, v.52, n.11, p.60-67, 2009.

RESNICK, M. O computador como pincel. In: VEJA. Limpeza de alto risco. Especial: um guia do mundo digital. São Paulo: Abril Cultural, n. 41, out. 2006.

SCAICO, P.D. et al. Ensino de Programação no Ensino Médio: Uma Abordagem Orientada ao Design com a linguagem Scratch. Braz. Jl. of Computers in Education, v.21, n.02, p.92, 2013. SCHULZE, K.G.; SHELBY, R.N.; TREACY, D.J.; WINTERSGILL, M.C. Andes: A Coached Learning Environment for Classical Newtonian Physics. In: Proceedings of the 11th International Conference on College Teaching and Learning. Jacksonville, FL, April, 2000.

VIGOTSKI, L.S. A formação social da mente: o desenvolvimento dos processos psicológicos superiores. 7. ed. São Paulo: Martins Fontes, 2007. Org. Michael Cole.

WANGENHEIM, C.G.; NUNES, V.R.; SANTOS,G.D. Ensino de Computação com SCRATCH no Ensino Fundamental. Revista Bras. de Informática na Educação, v.22, n.03, p. 115, 2014. WING, J.M. Computational thinking. Communications of the ACM, v.49, n.3, p. 33-35, 2006. 